\title{
Mycobacterium szulgai: A Rare Cause of Non-Tuberculous Mycobacteria Disseminated Infection
}

\author{
Ana Luisa Nunes ${ }^{\mathrm{a}, \mathrm{b}, \mathrm{f}}$, Ana Coimbra ${ }^{\mathrm{a}, \mathrm{b}}$, Ruben Carvalho, ${ }^{\mathrm{a}, \mathrm{c}}$, Carolina Figueiredo ${ }^{\mathrm{a}, \mathrm{d}}$, \\ Vania Almeida ${ }^{\text {a, e }}$, Jandira Lima ${ }^{\text {a, b}}$, Rui M. Santos ${ }^{\text {a, b }}$
}

\begin{abstract}
Mycobacterium szulgai (MS) is a rare and slow-growing type of nontuberculous mycobacteria (NTM), with a human isolation prevalence of less than $0.2 \%$ of all NTM cases. MS may cause pulmonary infection, extra-pulmonary localized disease involving the skin, lymph nodes, bone, synovial tissue or kidneys and disseminated infection, when two or more organs are affected. When disseminated infection is present, the patients usually have an underlying immunosuppressive condition. The authors report the case of a 25 -year-old patient with systemic lupus erythematosus, presenting with recurrent fever, non-productive coughing, weight loss and asthenia, as well as two violaceous plaques with superficial ulceration in the gluteal region. MS was isolated from the bronchial lavage and skin biopsy cultures, confirming the rare disseminated form of MS infection. After 10 months of follow-up on isoniazid, rifampin, ethambutol and pyrazinamide, no signs of relapse were evident. To date, only 16 other cases of MS disseminated disease have been reported.
\end{abstract}

Keywords: Mycobacterium szulgai; Disseminated infection; Nontuberculous mycobacteria; Cutaneous infection

\section{Introduction}

Non-tuberculous mycobacteria (NTM) are free-living organisms, ubiquitous in the environment. They are present in natural and drinking waters as well as soil samples, and exposure to these

Manuscript submitted November 9, 2021, accepted January 6, 2022

Published online February 16, 2022

aPraceta Professor Mota Pinto, Coimbra 3004-561, Portugal

b Internal Medicine Department, Centro Hospitalar e Universitario de Coimbra EPE, Coimbra, Portugal

'Infectious Diseases Department, Centro Hospitalar e Universitario de Coimbra EPE, Coimbra, Portugal

${ }^{\mathrm{d} D e r m a t o l o g y}$ Department, Centro Hospitalar e Universitario de Coimbra EPE, Coimbra, Portugal

${ }^{\mathrm{e} A n a t o m i c a l ~ P a t h o l o g y ~ D e p a r t m e n t, ~ C e n t r o ~ H o s p i t a l a r ~ e ~ U n i v e r s i t a r i o ~ d e ~ C o-~}$ imbra EPE, Coimbra, Portugal

${ }^{f}$ Corresponding Author: Ana Luisa Nunes, Internal Medicine Department, Centro Hospitalar e Universitario de Coimbra EPE, Coimbra, Portugal.

Email: ana.luisa.s.nunes@gmail.com

doi: https://doi.org/10.14740/jmc3885 reservoirs is thought to be the source of human infection, since human-to-human transmission has rarely been reported. At least 160 species of NTM have been identified, previously considered to be non-pathogenic. Since the second half of 20th century, they have been gradually recognized as potentially pathogenic organisms, causing a broad spectrum of disease manifestations [1].

Mycobacterium szulgai (MS) is a rare, slow-growing scotochromogen pathogen, accounting for less than $0.2 \%$ of NTM isolated strains. MS, named after the microbiologist T. Szulga (who developed the lipid analysis method to identify this pathogen), most commonly manifests as a pulmonary infection, clinically and radiologically undistinguishable from active $M y$ cobacterium tuberculosis infection. Extra-pulmonary manifestations have been reported involving the skin, lymph nodes, bone, synovial tissue and kidneys. Although MS infection frequently affects immunocompromised patients, disseminated infection is unusual. Given its low prevalence, no standardized treatment is available. Since MS appears to be susceptible in vitro to most of the antituberculous drugs, the few reports of a successful treatment involve combination therapy with multiple agents [2].

\section{Case Report}

\section{Investigations}

A 25-year-old female patient, graduating in veterinary medicine, presented with a low-grade recurrent fever, first noticed 12 months before, usually lasting for 3 to 6 days, mainly in the evening, associated with non-productive coughing, weight loss ( $15 \%$ of her previous weight) and asthenia, for the last 6 months. She had also developed tender erythematous subcutaneous nodules in her lower limbs compatible with erythema nodosum (Fig. 1a), as well as two violaceous plaques with superficial ulceration and a purulent exudate in the left gluteal region (Fig. 1b). Of note, she had a previous diagnosis of systemic lupus erythematosus (SLE) but she was not under follow-up. Until 3 months before admission, she had been on prednisolone $7.5 \mathrm{mg} /$ day. On physical examination, a right anterior cervical non-painful fibroelastic enlarged lymph node was also palpable.

\section{Diagnosis}

Laboratory tests revealed increased inflammatory parameters 


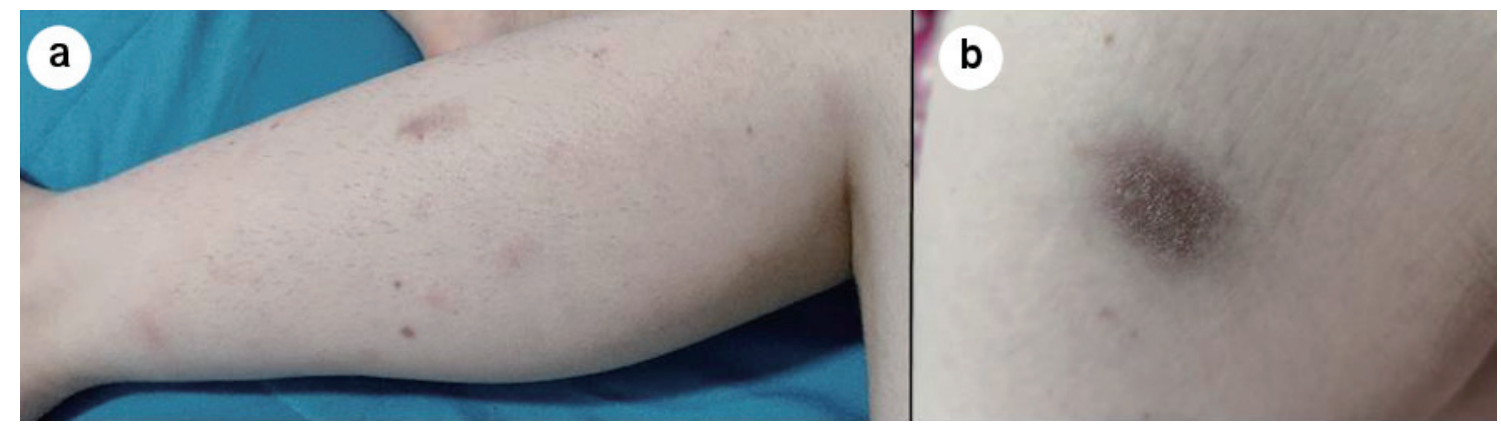

Figure 1. Cutaneous manifestations. (a) Erythematous nodules on the left lower limb. (b) Violaceous plaque in the left gluteal region.

and elevated cholestatic enzymes (Table 1). Additional blood cultures and multiple serologies were performed and reported as negative (Table 2).

Several mediastinal and cervical lymph nodes were enlarged in the chest computed tomography scan, some with a heterogeneous internal texture (Fig. 2). The positron emission tomography showed hypermetabolic activity in the lung parenchyma with a diffuse micronodular pattern, as well as in several supradiaphragmatic lymph nodes and two left gluteal subcutaneous nodular lesions. An excisional cervical lymph node biopsy showed a non-necrotizing granulomatous lymphadenitis. A bronchoscopy was executed, showing diffuse mucosal swelling and redness, as well as a bronchial biopsy, revealing two non-caseating epithelioid granulomas (Fig. 3). MS was isolated after 19 days from a liquid medium-based culture of the bronchial lavage. A skin biopsy of a gluteal lesion was performed, showing a neutrophilic infiltrate and suppurative granulomas with peripheral infiltration of histio- cytes, lymphocytes and plasmocytes (Fig. 4). MS was isolated in the skin culture after 23 days, confirming dissemination infection.

\section{Treatment}

The patient was started on an antituberculous regimen (ATR) according to her low body weight (isoniazid $300 \mathrm{mg}$ /day, rifampin $500 \mathrm{mg} /$ day, ethambutol $1,200 \mathrm{mg}$ /day, pyrazinamide $1,500 \mathrm{mg} /$ day).

\section{Follow-up and outcomes}

After 2 months on ATR, she had gained weight, she was reporting no fever, coughing or asthenia and there was no recurrence of the erythema nodosum. The MS disseminated infection was

Table 1. Blood Tests Results

\begin{tabular}{ll}
\hline Blood test & Result (normal range) \\
\hline Erythrocyte sedimentation rate & $63 \mathrm{~mm} / \mathrm{h}(1-20 \mathrm{~mm} / \mathrm{h})$ \\
Hemoglobin & $11.6 \mathrm{~g} / \mathrm{dL}(12-16 \mathrm{~g} / \mathrm{dL})$ \\
Mean corpuscular volume & $78 \mathrm{fL}(80-100 \mathrm{fL})$ \\
C-reactive protein & $12 \mathrm{mg} / \mathrm{dL}(0.5-1 \mathrm{mg} / \mathrm{dL})$ \\
Lactate dehydrogenase & $332 \mathrm{U} / \mathrm{L}(<247 \mathrm{U} / \mathrm{L})$ \\
Alkaline phosphatase & $316 \mathrm{U} / \mathrm{L}(30-120 \mathrm{U} / \mathrm{L})$ \\
Gamma-glutamyl transferase & $119 \mathrm{U} / \mathrm{L}(<31 \mathrm{U} / \mathrm{L})$ \\
Serum angiotensin converting enzyme & $26 \mathrm{U} / \mathrm{L}(8-52 \mathrm{U} / \mathrm{L})$ \\
Serum protein immunoelectrophoresis & Normal \\
Serum immunofixation & Normal \\
Ratio of kappa and lambda light chains & Normal \\
IgM, IgG, IgA, IgE & Normal \\
C3, C4 & Normal \\
Antinuclear antibodies & Positive $(1: 640$, dense fine speckled pattern $)$ \\
Anti-double stranded DNA & $0.5 \mathrm{IU} / \mathrm{mL}(>10 \mathrm{IU} / \mathrm{mL})$ \\
Extractable nuclear antigens antibodies & Negative \\
\hline
\end{tabular}

Ig: immunoglobulin; C: complement. 
Table 2. Serologies Results

\begin{tabular}{ll}
\hline Microorganism & Results \\
\hline Cytomegalovirus & Negative (IgM and $\operatorname{IgG})$ \\
Herpes simplex virus 1 and 2 & Negative (IgM and $\operatorname{IgG})$ \\
Epstein Barr virus & Negative (IgM, IgG and EBNA) \\
Hepatitis B virus & Negative (HBsAg, HBsAb and HBcAb) \\
Hepatitis C virus & Negative (IgM and $\operatorname{IgG})$ \\
Hepatitis A virus & Negative (IgM and $\operatorname{IgG})$ \\
Human immunodeficiency virus & Negative (Ag/Ab) \\
Treponema pallidum & Negative (IgM and $\operatorname{IgG})$ \\
Toxoplasma gondii & Negative (IgM and $\operatorname{IgG})$ \\
Rickettsia conorii & Negative (IgM and $\operatorname{IgG})$ \\
Coxiella burnetii & Negative (IgM and $\operatorname{IgG})$ \\
Borrelia burgdorferi & Negative (IgM and $\operatorname{IgG})$ \\
Brucella species & Negative (IgM and $\operatorname{IgG})$ \\
Bartonella henselae & Negative (IgM and $\operatorname{IgG})$ \\
\hline
\end{tabular}

Ig: immunoglobulin; EBNA: Epstein-Barr nuclear antigen; HbsAg: hepatitis B surface antigen; HbsAb: hepatitis B surface antibody; HbcAb: hepatitis $B$ core antibody; Ag: antigen; Ab: antibody.

interpreted as being markedly improved with the chosen ATR. As her SLE disease activity index was found to be unchanged compared to past values, the addition of oral prednisolone on the patient's previous dosage $(7.5 \mathrm{mg} /$ day $)$ was postponed until at least 12 months on ATR have been achieved. After 10 months of follow-up no signs of relapse were evident, the patient was found to be asymptomatic and no analytical or imaging tests were suggestive of treatment failure.

\section{Discussion}

MS is a slow-growing NTM, first reported in 1972, which has the ability to grow in low carbon and oxygen concentrations [3]. MS is characterized by a thin peptidoglycan layer surrounded by a thick lipid-rich outer membrane, increasing its survivability in multiple environments. Additionally, the hydrophobic cell sur-

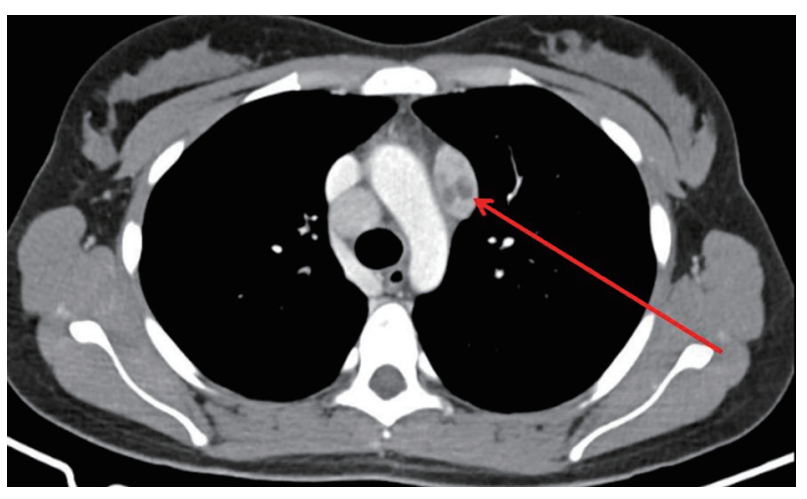

Figure 2. Enlarged mediastinal lymph node with a heterogeneous internal texture (arrow) on the computed tomography (CT) chest scan. face enables resistance to disinfectants and antibiotics [1].

MS is ubiquitous in municipal water supplies, making human exposure common and asymptomatic colonization of the respiratory tract possible. Despite this, MS is rarely isolated in humans. MS-induced disease is usually not reported to public health authorities, leading to sparse epidemiological and surveillance data [4].

When MS is identified in extra-pulmonary tissues, even without associated symptoms, the probability of true infection is high [5]. Contrasting with Mycobacterium tuberculosis, when MS is identified in lung tissue, it may not be pathogenic, but a non-significant colonization. When respiratory symptoms are also present, MS is generally the causative agent and adequate treatment and careful follow-up are required [6]. Since no specific diagnostic standards for MS disease have been established, the general guidance provided

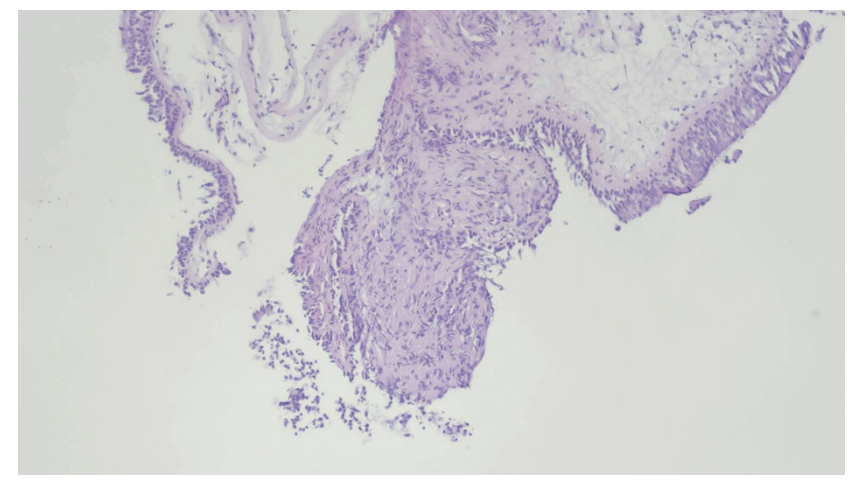

Figure 3. Two noncaseating epithelioid granulomas with tightly packed epithelioid cells and overlapped lymphocytes. Respiratory epithelium with normal morphology. (hematoxylin and eosin staining, $\times 100$ magnification). 


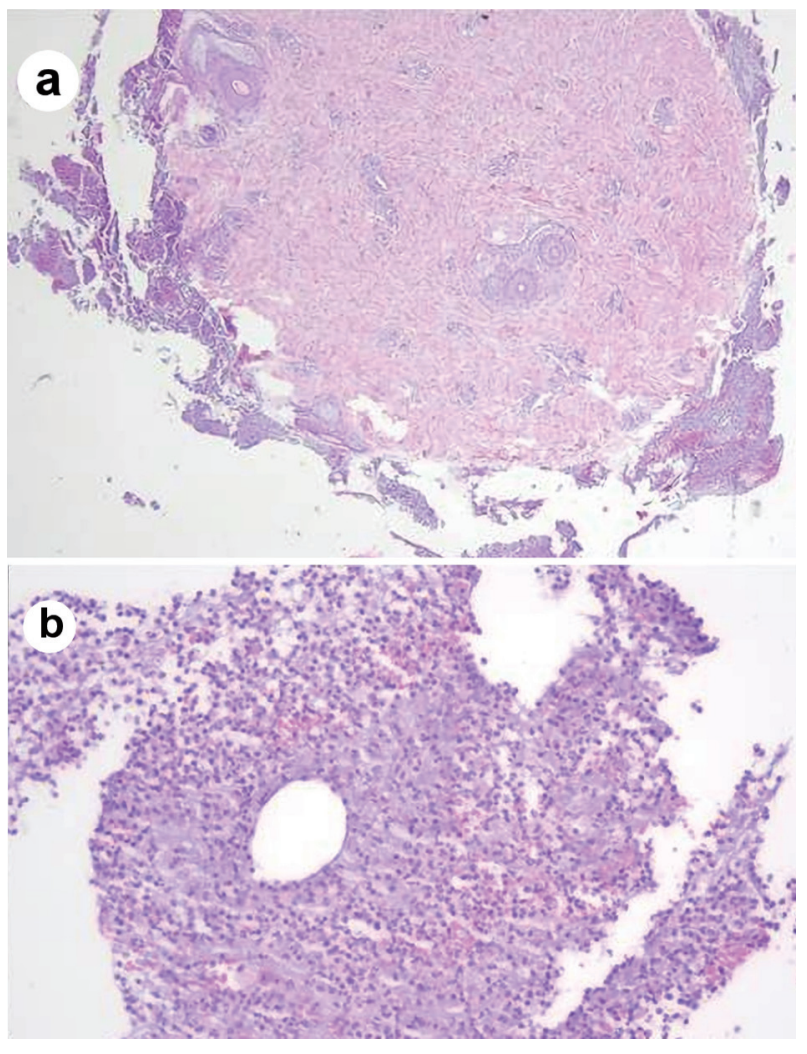

Figure 4. Skin biopsy showing neutrophilic infiltrate and focal granulomas (a) composed of histiocytes, lymphocytes and plasmacytes (b).

by the revised 2020 NTM disease guidelines by the Infectious Disease Society of America and the European Society of Clinical Microbiology and Infectious Diseases may be useful for clinicians. Of note, extra-pulmonary NTM infection and specific guidance for MS disease are not included [7].

MS may cause pulmonary and extra-pulmonary disease, as well as disseminated infection, with more than one organ affected. To date, only 16 cases of disseminated disease have been reported, predominantly in young male patients, and only $50 \%$ of these cases achieved a complete cure. In the few reported cases of disseminated infection, the most commonly affected location was the bone, followed by the skin and the lymph nodes. Despite being traditionally considered a low virulence pathogen, MS appears to cause disseminated infection in patients with immunosuppressive conditions or taking immunosuppressive drugs [8]. Our patient's SLE was interpreted as the likely immunosuppressive condition.

MS is reported to be susceptible to most antituberculous agents, but no optimal regimen or duration of treatment is established. A few case reports suggest the use of at least three drugs such as isoniazid, rifampin and ethambutol for at least 12 months. Other active drugs include clarithromycin and moxifloxacin [9]. NTM are not sensitive to pyrazinamide, and its use in this case may not have contributed to the treatment efficacy, considering the other three active drugs against MS. When there is no improvement with medical treatment alone and the disease is localized, resection of the diseased portion of the involved organ may be considered.
Our patient developed the rare disseminated form of MS infection, affecting both the lungs and the skin (confirmed by bronchial lavage and skin biopsy cultures). The MS treatment was started with a weight-adjusted regimen of at least three active drugs and after a 10-month follow-up the treatment is succeeding, the patient is asymptomatic, showing resolution of the skin lesions, normalization of the inflammatory parameters and no radiological evidence of relapsing disease.

The authors hope to offer insight of the possibility of disseminated MS infection in young patients presenting with multiple organ involvement and an underlying immunosuppressive condition.

\section{Learning points}

Mycobacterium szulgai is ubiquitous in the environment, leading to a common human exposure but a human isolation prevalence of less than $0.2 \%$ of all non-tuberculous mycobacteria cases. Mycobacterium szulgai usually causes pulmonary infection but may involve the bone, the skin and the lymph nodes. Disseminated infection is very rare and is associated with an immunocompromised status.

\section{Acknowledgments}

None to declare.

\section{Financial Disclosure}

None to declare.

\section{Conflict of Interest}

None to declare.

\section{Informed Consent}

Patient's informed consent for publication of this report was obtained, including any accompanying photographs.

\section{Author Contributions}

Ana Luisa Nunes, Ana Coimbra, Ruben Carvalho and Carolina Figueiredo contributed to the writing of this manuscript; all the authors were involved in the patient's treatment and in the review of this report.

\section{Data Availability}

The data supporting the findings of this study are available from the corresponding author upon reasonable request. 


\section{Abbreviations}

ATR: antituberculous regimen; MS: Mycobacterium szulgai; NTM: non-tuberculous mycobacteria; SLE: systemic lupus erythematosus

\section{References}

1. Daley CL, Griffith DE. Nontuberculous Mycobacterial Infections. In: Courtney Broaddus V, Mason RJ, Ernst JD, King TE, Lazarus SC, Murray JF, et al, editors. Murray and Nadel's Textbook of Respiratory Medicine. 6th ed. Canada: W.B. Saunders; 2016. p. 629-645.e6.

2. Gido RDS, Wojciechowski AL, Bajwa RP. Pulmonary infection with Mycobacterium szulgai: A case report. SAGE Open Med Case Rep. 2019;7:2050313X18823448.

3. Marks J, Jenkins PA, Tsukamura M. Mycobacterium szulgai - a new pathogen. Tubercle. 1972;53(3):210-214.

4. Kendall BA, Winthrop KL. Update on the epidemiology of pulmonary nontuberculous mycobacterial infections.
Semin Respir Crit Care Med. 2013;34(1):87-94.

5. Ruderman EM, Flaherty JP. Mycobacterial Infections of Bones and Joints. In: Firestein GS, Budd RC, Gabriel SE, McInnes IB, O'Dell JR, editors. Kelley and Firestein's Textbook of Rheumatology. 10th ed. China: Elsevier; 2017. p. 1905-1917.

6. van Ingen $\mathrm{J}$, Boeree MJ, de Lange $\mathrm{WC}$, de Haas PE, Dekhuijzen PN, van Soolingen D. Clinical relevance of Mycobacterium szulgai in The Netherlands. Clin Infect Dis. 2008;46(8):1200-1205.

7. Daley CL, Iaccarino JM, Lange C, Cambau E, Wallace RJ, Jr., Andrejak C, Bottger EC, et al. Treatment of nontuberculous mycobacterial pulmonary disease: an official ATS/ERS/ESCMID/IDSA clinical practice guideline. Eur Respir J. 2020;56(1):2000535.

8. Weng TP, Syue LS, Lee NY. Disseminated Mycobacterium szulgai infection in a patient with anti-interferongamma autoantibodies. IDCases. 2020;21:e00848.

9. Basille D, Jounieaux V, Andrejak C. Treatment of Other Nontuberculous Mycobacteria. Semin Respir Crit Care Med. 2018;39(3):377-382. 